\title{
Combined Expression of Metastasis Related Markers Naa10p, SNCG and PRL-3 and its Prognostic Value in Breast Cancer Patients
}

\author{
Li Min ${ }^{1}$, Ruo-Lan Ma², Hua Yuan ${ }^{3}$, Cai-Yun Liu', Bing Dong ${ }^{4}$, Cheng Zhang ${ }^{1}$, \\ Yan Zeng ${ }^{1}$, Li Wang ${ }^{1}$, Jian-Ping Guo ${ }^{1}$, Li-Ke Qu ${ }^{1}$, Cheng-Chao Shou ${ }^{1 *}$
}

\begin{abstract}
Combinations of multiple biomarkers representing distinct aspects of metastasis may have better prognostic value for breast cancer patients, especially those in late stages. In this study, we evaluated the protein levels of $\mathrm{N}$ - $\alpha$-acetyltransferase 10 protein (Naa10p), synuclein- $\gamma$ (SNCG), and phosphatase of regenerating liver-3 (PRL3 ) in 365 patients with breast cancer by immunohistochemistry. Distinct prognostic subgroups of breast cancer were identified by combination of the three biomarkers. The Naa10p+SNCG-PRL-3- subgroup showed best prognosis with a median distant metastasis-free survival (DMFS) of 140 months, while the Naa10p-SNCG+PRL-3+ subgroup had the worst prognosis with a median DMFS of 60.5 months. Multivariate analysis indicated Naa10p, SNCG, PRL-3, and the TNM classification were all independent prognostic factors for both DMFS and overall survival (OS). The three biomarker combination of Naa10p, SNCG and PRL-3 performed better in patients with lymph node metastasis, especially those with more advanced tumors than other subgroups. In conclusion, the combined expression profile of Naa10p, SNCG and PRL-3, alone or in combination with the TNM classification system, may provide a precise estimate of prognosis of breast cancer patients.
\end{abstract}

Keywords: Combined expression - Naa10p - SNCG - PRL-3 - prognosis - breast cancer

Asian Pac J Cancer Prev, 16 (7), 2819-2826

\section{Introduction}

Breast cancer is not only the most frequently diagnosed cancer in women but also the leading cause of cancer death among females worldwide (Jemal et al., 2011). Breast cancer accounts for $29 \%$ of the total cancer cases and $14 \%$ of the cancer deaths (Jemal et al., 2011; Siegel et al., 2012). Recently, most breast cancer of early stages can be cured by radical mastectomy, and nearly $90 \%$ breast cancer patients can survive more than 5 years with multimodality treatment (Holmes et al., 2010; Saini et al., 2011). However, in developing countries, death rate of breast cancer is still above $40 \%$, and most of the deaths were caused by metastasis (Jemal et al., 2011; Rahimzadeh et al., 2014). Micrometastasis is hard to predict by all the current prognosis means alone, which makes patients with the same clinical stages always show different survival time (Cox et al., 2011; Jafferbhoy and McWilliams, 2012), suggesting that novel biomarkers and updated staging procedures should be developed to provide precise cancer prognosis. In this study, we proposed that combining markers representing different aspects of breast cancer metastasis could provide a better prognostic value.
$\mathrm{N}-\alpha$-acetyltransferase 10 protein (Naa10p), the catalytic subunit of $\mathrm{N}$-acetyltransferase A (NatA), is involved in cell cycle (Lim et al., 2006), proliferation (Lim et al., 2006; Seo et al., 2010), apoptosis (Gromyko et al., 2010), autophage (Kuo et al., 2010), cell motility (Bauer et al., 2009; Hua et al., 2011), neuron development (Ohkawa et al., 2007; Ohkawa et al., 2008) and 28S proteasome activity (Min et al., 2013). Naa10p is overexpressed in various types of cancer (Lee, 2010; Hua et al., 2011), and its overexpression in breast cancer is negatively correlated with metastasis and indicates good prognosis through different molecular pathway (Bauer et al., 2009; Hua et al., 2011; Zeng et al., 2013).

Synuclein-gamma (SNCG), also named breast cancer-specific gene, been specifically implicated in neurodegenerative diseases such as Alzheimer's disease (AD) and Parkinson's disease (PD) (Polymeropoulos et al., 1997). Expression of SNCG in breast cancer cells impairs cell cycle checkpoint (Inaba et al., 2005), promotes chemoresistance (Singh et al., 2007), enhances metastasis in nude mice (Jia et al., 1999), while its mechanism is not fully understood. SNCG was found overexpressed in various types of cancer and its overexpression predicted

${ }^{I}$ Department of Biochemistry and Molecular Biology, Key Laboratory of Carcinogenesis and Translational Research (Ministry of Education), ${ }^{2}$ Department of Thoracic Surgery, ${ }^{3}$ Breast Center, ${ }^{4}$ Department of Pathology, Peking University Cancer Hospital and Institute, Beijing, China *For correspondence: scc@bjcancer.org 
poor prognosis of both ovarian cancer and breast cancer (Czekierdowski et al., 2006; Guo et al., 2007; Liu et al., 2010; Wu et al., 2013).

Phosphatase of regenerating liver-3 (PRL-3) is also a metastasis-associated gene. PRL-3 is an important and comprehensive cell function regulator, involved in cell cycle, proliferation (Polato et al., 2005), invasion and metastasis (Zheng et al., 2010). Overexpression of PRL-3 promoted invasion and metastasis of cancer cells (Amsterdam et al., 2012; Guzinska-Ustymowicz et al., 2013; Guzinska-Ustymowicz et al., 2011). Patients with increased PRL-3 level showed different outcomes in different types of cancer (Al-Aidaroos and Zeng, 2010). Previous study indicated that PRL-3 overexpression resulted in poor prognosis of breast cancer patients (Wang, 2006).

Naa10p, SNCG and PRL-3 are three critical factors involved in the regulation of breast cancer carcinogenesis. According to the recent information, these three proteins function through different molecular mechanisms in the regulation of breast cancer metastasis. Combination of these three biomarkers and an integrated evaluation of them may represent a more comprehensive view of breast caner, which could provide an opportunity to investigate and explore a modified staging system for breast caner patients. In this study, we investigated the possible correlations of these three proteins with clinical outcome, in an effort to identify high metastasis risk patients with breast cancer to make better treatment options for them.

\section{Materials and Methods}

\section{Patients}

Breast cancer tissue specimens were obtained from 365 women having breast surgery (radical or modified radical mastectomy) at Peking University Cancer Hospital and Institute between 1996 and 2002. The patients' age ranged from 25 to 81 (with a median of 50 years). The tumors were staged based on Union Internationale Contre Le Cancer (UICC) TNM classification: 141 Stage I tumors,

Table 1. Influences of Clinicopathologic Factors, Naa10p, SNCG, PRL-3 and their Combination on Metastasis in Breast Cancer Patients

\begin{tabular}{|c|c|c|c|c|}
\hline Characteristics & No. of Cases & No. of Distant Metastasis & HR $(95 \%$ CI $)$ & $p$ value \\
\hline$\overline{\text { Age }}$ & & & & 0.66 \\
\hline$<50$ years & 168 & 56 & 1 & \\
\hline$\geq 50$ years & 197 & 70 & $1.102(0.715-1.701)$ & \\
\hline Tumor size & & & & $<0.001$ \\
\hline$\leq 2 \mathrm{~cm}$ & 213 & 55 & 1 & \\
\hline$>2 \mathrm{~cm}$ & 152 & 71 & $2.518(1.618-3.919)$ & \\
\hline Lymph node status & & & & $<0.001$ \\
\hline negative & 206 & 35 & 1 & \\
\hline positive & 159 & 91 & $6.538(4.044-10.572)$ & \\
\hline TNM stage & & & & $<0.001$ \\
\hline I & 141 & 20 & 1 & \\
\hline II & 128 & 34 & $2.188(1.184-4.046)$ & \\
\hline III+IV & 96 & 72 & $18.150(9.369-35.160)$ & \\
\hline ER & & & 0.217 & \\
\hline negative & 121 & 46 & 1 & \\
\hline positive & 232 & 73 & $0.749(0.473-1.186)$ & \\
\hline PR & & & 0.121 & \\
\hline negative & 169 & 64 & 1 & \\
\hline positive & 183 & 55 & $0.705(0.453-1.098)$ & \\
\hline HER2 & & & & 0.424 \\
\hline negative & 222 & 69 & 1 & \\
\hline positive & 69 & 25 & $1.260(0.714-2.222)$ & \\
\hline Naa10p & & & & 0.004 \\
\hline negative & 291 & 111 & 1 & \\
\hline positive & 74 & 15 & $0.412(0.223-0.762)$ & \\
\hline SNCG & & & & $<0.001$ \\
\hline negative & 237 & 60 & 1 & \\
\hline positive & 128 & 66 & $3.140(1.995-4.944)$ & \\
\hline PRL-3 & & & & 0.051 \\
\hline negative & 236 & 73 & 1 & \\
\hline positive & 129 & 53 & $1.557(0.997-2.433)$ & \\
\hline Combined Naa10p/SNCG & & & $<0.001$ & \\
\hline Naa10p-SNCG+ & 94 & 57 & 1 & \\
\hline Naa10p+SNCG- & 40 & 6 & $0.115(0.044-0.300)$ & \\
\hline Others & 231 & 63 & $0.242(0.147-0.403)$ & \\
\hline Combined Naa10p/SNCG/PRL-3 & & & $<0.001$ & \\
\hline Naa10p-SNCG+PRL-3+ & 42 & 27 & 1 & \\
\hline Naa10p+SNCG-PRL-3- & 27 & 6 & $0.159(0.053-0.479)$ & \\
\hline others & 296 & 93 & $0.255(0.129-0.501)$ & \\
\hline
\end{tabular}

*HR, hazard ratio; CI, confidence interval 
128 Stage II tumors and 96 Stage III/IV tumors. The presence of lymph node metastasis had been determined by histological examination: 206 negative and 159 positive. All of the patients were followed up by interview in clinic or phone call. The total period of follow-up was 60-192 months with a median of 127 months. The study was approved and supervised by the Medical Ethics Committee of Peking University Cancer Hospital and Institute. Participants attending this study were informative for the well-characterized clinicopathologic variables, including patient outcome. All the specimens were preserved by Department of Pathology, fixed and paraffin-embedded for routine immunohistochemistry analysis. Overall survival (OS) time was calculated from the date of surgery to the date of death due to any cause. Distant metastasis-free survival (DMFS) time was calculated for patients from the date of surgery to the date of distant metastasis. Data on patients, who had survived until the end of follow-up period, were censored at the date of last contact. The clinicopathologic characteristics of patients were summarized in Table 1 .

\section{Immunohistochemistry}

Immunohistochemical staining procedure was described in our previous paper (Wang et al., 2008). All paraffin-embedded specimens were cut into $5 \mu \mathrm{m}$ sections. After baking at $60^{\circ} \mathrm{C}$ overnight, sections were dewaxed and rehydrated through xylene and an alcohol series. Thereafter, antigen retrieval was performed via microwave cooking in ethylene diamine tetraacetic acid $(\mathrm{pH} \mathrm{8.0,}$ Zymed) for $20 \mathrm{~min}$. Endogenous peroxidase activity was blocked by incubation in $3 \%$ hydrogen peroxide for $10 \mathrm{~min}$ at room temperature. Non-specific binding was blocked with $10 \%$ goat serum. After that, anti-Naa10p, Anti-PRL-3 and anti-SNCG monoclonal antibodies, which were prepared in our laboratory and the quality, specificity, and sensitivity had been determined in Refs. (Zeng et al., 2013), (Wang et al., 2008) and (Guo et al., 2007), respectively, were applied to each slide and incubated at $4^{\circ} \mathrm{C}$ overnight. After 3 washes with phosphate-buffered saline with $0.1 \%$ Tween-20, the specimens were incubated with second antibody from the EnvisionTM kit (Dako Cytomation, Cambridge, UK) for $45 \mathrm{~min}$ at room temperature. The reaction product was visualized with diaminobenzidine (Sigma) for $5 \mathrm{~min}$ at room temperature and the sections were counterstained with hematoxylin. Normal mouse $\mathrm{IgG}$ was used as a negative control of the primary antibody.

\section{Evaluation of immunohistochemical staining}

The results were evaluated under light microscopy (APPLIED IMAGING at 200x) independently by two experienced pathologists without prior knowledge of the clinical information. The discrepancies $(<5 \%)$ were resolved by simultaneous re-evaluation. We assessed both the percentage of positive cells and the intensity of staining in 10 randomly chosen microscopic field. A semiquantitative scoring system in tumor cells was graded according to a 4-value classification scale as follows: area of staining as $<10 \%$ of all cancer cells stained within the section was graded as none (0), staining intensity
( $>10 \%$ of all cancer cells stained within the section) was graded as weak (1), moderate (2) or strong (3). The immunohistochemical evaluation of Naa10p, SNCG and PRL-3 were presented as either "negative" or "positive", in which score of $\geq 2$ was defined as "positive".

\section{Statistical analysis}

Correlations between protein expression levels and patient clinicopathologic characteristics were performed using Pearson $\chi^{2}$ test. The Kaplan-Meier method was used to estimate DMFS and OS rates, and the survival differences were tested by log-rank method. The Cox proportional hazard model was used for multivariate analysis to investigate the independence of the risk factors identified as significant in the univariate analysis. Hazard ratios $(\mathrm{HR})$ and $95 \%$ confidence interval $(\mathrm{CI})>1.0$ indicate that positive expression is associated with a poor prognosis while $<1.0$ indicate that positive expression is associated with a good prognosis. Survival analysis stratified by TNM classification and lymph node metastasis status were also conducted. All statistical analyses were 2-sided, and comparisons made in which $p$ values less than 0.05 were considered statistically significant. All statistical analyses were performed using SPSS for Windows Software (version 13.0).

\section{Results}

Expression of Naa10p, SNCG, PRL-3 and their Correlation With the Presence of Distant Metastases

Immunohistochemistry (IHC) of Naa10p, SNCG and PRL-3 was conducted using 365 breast cancer tissue specimens. The positive rate of Naa10p, SNCG and PRL-3 in breast cancer were $20.3 \%$ (74/365), 35.1\% (128/365) and $35.3 \%$ (129/365), respectively. Spearman correlation analysis showed that SNCG had a weak correlation with both Naa10p ( $\mathrm{r}=0.115, p=0.028)$ and PRL-3 $(\mathrm{r}=0.153$, $p=0.003$ ), and Naa10p showed no association with PRL3. Naa10p weakly correlated with lymph node metastasis ( $\mathrm{r}=-0.127, p=0.015)$, while SNCG weakly correlated with lymph node metastasis $(\mathrm{r}=0.246, p<0.001)$, tumor size 
Table 2. Prognostic Value of Clinicopathologic Factors, Naa10p, SNCG PRL-3 and their Combination on Distant Metastasis-free Survival (DMFS) and Overall Survival (OS) of 365 Patients with Breast Cancer

\begin{tabular}{|c|c|c|c|c|}
\hline \multirow[b]{2}{*}{ Characteristics } & \multicolumn{2}{|c|}{ DMFS } & \multicolumn{2}{|c|}{ OS } \\
\hline & HR $(95 \% \mathrm{CI})$ & $p$ value & HR $(95 \%$ CI) & $p$ value \\
\hline Tumor size & & $<0.001$ & & $<0.001$ \\
\hline$\leq 2 \mathrm{~cm}$ & 1 & 1 & & \\
\hline$>2 \mathrm{~cm}$ & $2.159(1.517-3.072)$ & & $2.333(1.545-3.525)$ & \\
\hline Lymph node status & & $<0.001$ & & $<0.001$ \\
\hline negative & 1 & 1 & & \\
\hline positive & $4.714(3.187-6.974)$ & & $5.084(3.174-8.144)$ & \\
\hline TNM stage & & $<0.001$ & & $<0.001$ \\
\hline I & 1 & 1 & & \\
\hline II & $1.965(1.130-3.419)$ & & $1.957(0.990-3.870)$ & \\
\hline III+IV & $9.787(5.937-16.134)$ & & $10.030(5.483-18.349)$ & \\
\hline 0.002 & 0.003 & & & \\
\hline negative & 1 & 1 & & \\
\hline positive & $0.434(0.253-0.745)$ & & $0.346(0.174-0.689)$ & \\
\hline SNCG & & $<0.001$ & & $<0.001$ \\
\hline negative & 1 & 1 & & \\
\hline positive & $2.683(1.887-3.815)$ & & $2.772(1.843-4.171)$ & \\
\hline PRL-3 & & 0.025 & 0.018 & \\
\hline negative & 1 & & $1.639(1.090-2.464)$ & \\
\hline positive & $1.502(1.053-2.142)$ & & & \\
\hline Combined Naa10p/SNCG & & $<0.001$ & & $<0.001$ \\
\hline Naa10p-SNCG+ & 1 & 1 & & \\
\hline Naa10p+SNCG- & $0.140(0.060-0.328)$ & & $0.130(0.046-0.362)$ & \\
\hline others & $0.317(0.221-0.456)$ & & $0.287(0.189-0.435)$ & \\
\hline Combined Naa10p/SNCG/PRL-3 & & $<0.001$ & & $<0.001$ \\
\hline Naa10p-SNCG+PRL-3+ & 1 & 1 & & \\
\hline Naa10p+SNCG-PRL-3- & $0.180(0.074-0.440)$ & & $0.166(0.057-0.484)$ & \\
\hline others & $0.333(0.216-0.512)$ & & $0.315(0.196-0.507)$ & \\
\hline
\end{tabular}

$(\mathrm{r}=0.194, p<0.001)$ and TNM classification $(\mathrm{r}=0.242$, $p<0.001)$. Besides these, no correlation was found between any of the three molecular markers and other traditional clinical and pathological features.

The associations between these factors and distant metastasis were also analyzed. As expected, clinicopathologic features, including TNM stage (III/IV, II versus I; $p<0.001)$, lymph node metastasis $(p<0.001)$ and tumor size $(p<0.001)$ were significantly related with distant metastasis of breast cancer, whereas age, ER, PR and HER2 status did not affect distant metastasis ( $p>0.05$; Table 1). Among the 3 molecular markers, Naa10p significantly negatively correlated with the presence of distant metastases $(p=0.004)$, and SNCG significantly positively correlated with the presence of distant metastases $(p<0.001)$. PRL-3 positive subgroup showed a HR of 1.557 compared to the PRL-3 negative subgroup, but the $p$ value was not statistically significant $(p=0.051)$. The correlation with three markers with metastasis was reasonable considering their molecular function in cancer cell motility (Hibi et al., 2009; Peng et al., 2009; Zeng et al., 2013). Consistent with the above, multimarker phenotype of Naa10p/SNCG $(p<0.001)$ and of Naa10p/SNCG/PRL-3 $(p<0.001)$ also correlated with the presence of distant metastases (Table 1).

Prognostic Value of Multimarker System for Patients With Breast Cancer

The prognostic value of the Naa10p, SNCG and PRL3 alone and multimarker combination was evaluated.
Kaplan-Meier curves and corresponding $p$ values from the log-rank test showed the effect of each marker on DMFS time (Figure 1). Obviously, Naa10p positive patients showed a significantly longer DMFS time than the Naa10p negative group (Figure $1 \mathrm{~A} ; \log$ rank $\chi^{2}=5.141$; $p=0.023)$. However, patients with SNCG positive tumors had a significantly shorter DMFS time than those with SNCG negative tumors (Figure $1 \mathrm{~B}$; $\log$ rank $\chi^{2}=32.87$; $p<0.001)$. Same conclusion was obtained for PRL-3 (Figure 1C; log rank $\chi^{2}=32.87 ; p<0.001$ ). When combined Naa10p with SNCG, Naa10p+SNCG- subgroup had a median DMFS time of 133.5 months, longer than 122 months of Naa10p+SNCG+/Naa10p-SNCG- subgroup, while Naa10p-SNCG+ subgroup got the worst prognosis with a median DMFS time of 60.5 months (Figure $1 \mathrm{D} ; \log$ rank $\left.\chi^{2}=56.25 ; p<0.001\right)$. When adding PRL-3 expression to this model, the best prognosis subgroup (Naa10p+SNCG-PRL-3-) displayed a median DMFS time of 140 months, nearly four times of the worst prognosis subgroup (Naa10p-SNCG+PRL-3+) (Figure 1E; log rank $\left.\chi^{2}=32.53 ; p<0.001\right)$. We also classified all patients to ER/PR subgroup (ER+ or PR+), HER2 subgroup (ER-PR-HER2+) and triple negative (TN) subgroup (ER-PR-HER2-) according to clinical data (Figure 1F; $\log$ rank $\chi^{2}=3.97 ; p=0.138$ ). Compared with this, our new multimarker classification system performed better at the respect of prognosis.

For the evaluation of OS, Kaplan-Meier curves and log-rank test were also performed and the results are very similar to those of DMFS (Figure 2). Naa10p positive 

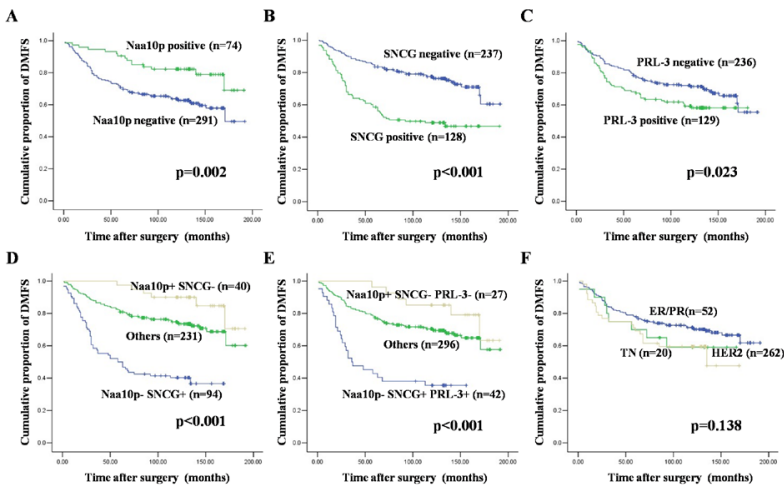

Figure 1. Kaplan-Meier Curve of DMFS in 365 Patients with Breast Cancer Evaluated According to Naa10p, SNCG, PRL-3 Level Alone and Their Combinations. Cases with Naa10p A), SNCG B), PRL-3 C), combined Naa10p/ SNCG D), combined Naa10p/SNCG/PRL3 E) and combined ER/PR/HER2- negative tumors (blue line) versus cases with the corresponding protein-positive tumors (green line)
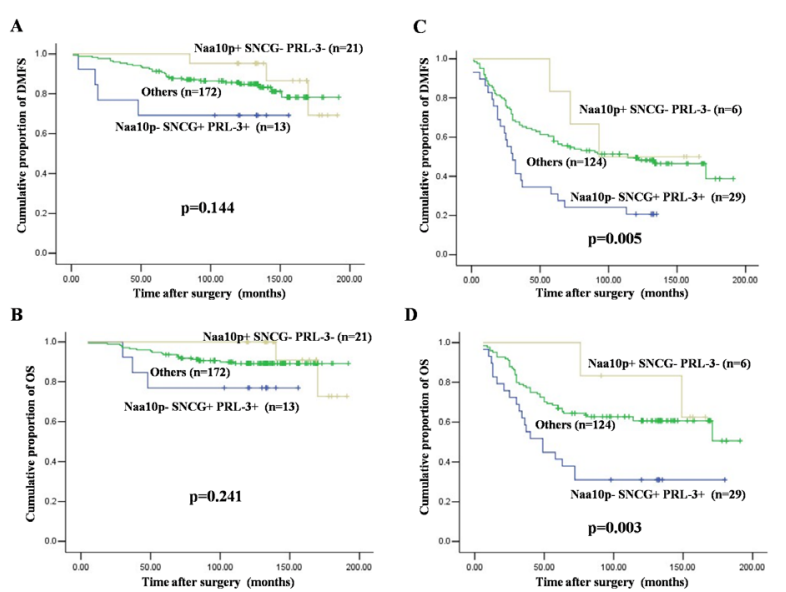

Figure 3. Kaplan-Meier Curve of DMFS and OS in 206 Patients with Breast Cancer with $\mathrm{LN}$ - and 159 with LN+ Evaluated According to Naa10p, SNCG, PRL-3 combination. A) DMFS of $\mathrm{LN}-$; B) DMFS of $\mathrm{LN}+$; C) OS of $\mathrm{LN}-$; D) OS of LN+

patients showed a significantly longer OS time than the Naa10p negative group (Figure 2A; $\log$ rank $\chi^{2}=10.05$; $p=0.002$ ), while $S N C G$ positive patients had a significantly shorter DMFS time than those with SNCG negative tumors (Figure 2B; $\log$ rank $\chi^{2}=26.23 ; p<0.001$ ). PRL-3 positive subgroup also displayed a shorter OS than PRL-3 negative subgroup (Figure 2C; log rank $\chi^{2}=5.79 ; p=0.016$ ). When combined Naa10p with SNCG, Naa10p+SNCG- subgroup showed the best prognosis while Naa10p-SNCG- showed the worst (Figure 2D; log rank $\chi^{2}=50.09 ; p<0.001$ ). Combining all three markers, Naa10p+SNCG-PRL-3subgroup displayed the longest median OS (Figure 2E; $\log$ rank $\left.\chi^{2}=29.47 ; p<0.001\right)$. The new three markers classification system performed better than the traditional biomarker classification system (ER/PR, HER2 and TN; Figure $2 \mathrm{~F}$; log rank $\left.\chi^{2}=0.66 ; p=0.719\right)$ in OS prediction.

Univariate Cox analysis was performed and its results were showed in Table 2. Subsequently, all factors that showed prognostic significance in the univariate Cox analysis were included in the multivariate Cox proportional hazard model. In multivariate analysis,
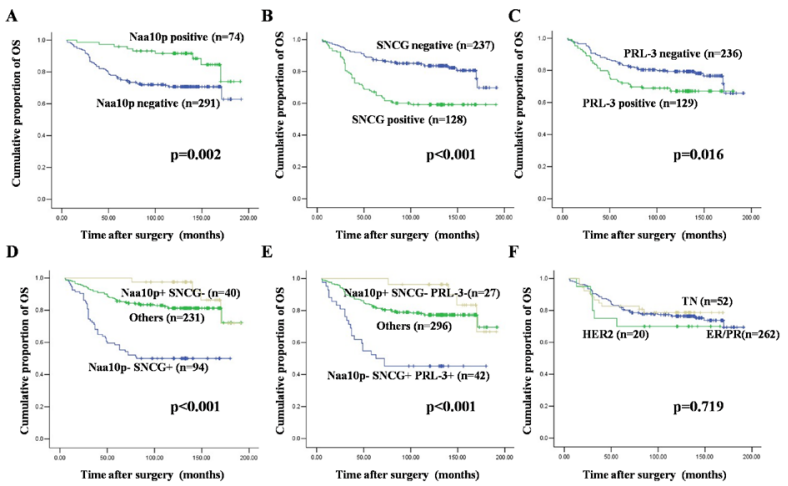

Figure 2. Kaplan-Meier Curve of OS in 365 Patients with Breast Cancer Evaluated According to Naa10p, SNCG, PRL-3 Level Alone and Their Combinations. Cases with Naa10p. A), SNCG B), PRL-3 C), combined Naa10p/SNCG D), combined Naa10p/SNCG/PRL3 E) and combined ER/PR/HER2- negative tumors (blue line) versus cases with the corresponding protein-positive tumors (green line)

Naa10p $[p=0.002 ; \mathrm{HR}(95 \% \mathrm{CI})=0.406(0.236-0.700)]$, SNCG $[p=0.002 ; \mathrm{HR}(95 \% \mathrm{CI})=2.058(1.429-2.963)]$, PRL-3 [ $p=0.002$; HR $(95 \% \mathrm{CI})=0.465$ (1.023-2.097)], and TNM classification all remained independent prognostic factors for DMFS (Table 3, middle column). Combined Naa10p/SNCG and combined Naa10p/SNCG/PRL-3 were both independent indicators only in univariate analysis, but not in multivariate analysis. Similar result was obtained in the analysis of OS (Table 3, right column).

Prognostic Value of Multimarker System for Patients with Breast Cancer Stratified by Lymph Node Metastasis

Subsequently, the prognostic value of the Naa10p/ SNCG/PRL-3 three biomarkers classification system was evaluated in the tumors stratified into subgroups with $(\mathrm{LN}+)$ or without (LN-) lymph node metastasis.

For the LN- patients, patients with Naa10p+SNCGPRL-3- tumors had a trend of longer DMFS compared with other subgroups, but did not reach a statistical difference (Figure $3 \mathrm{~A}$; $\log$ rank $\chi^{2}=3.88 ; p=0.144$ ), and similar results were gained for OS (Figure $3 \mathrm{~B}$; log rank $\chi^{2}=2.85 ; p=0.241$ ). In multivariate analysis, only SNCG remained independent prognostic factors for DMFS $[p=0.078 ; \mathrm{HR}(95 \% \mathrm{CI})=1.884(0.932-3.809)]$ and no variate was included in the model of OS.

For the $\mathrm{LN}+$ patients, Naa10p+SNCG-PRL-3subgroup displayed a longer median DMFS of 124 months and a longer median OS of 152 months, compared with 30 months DMFS and 49 months OS of Naa10pSNCG+PRL-3+ subgroup (Figure 3C, DMFS, log rank $\chi^{2}=10.67, p=0.005$; Figure 3D, OS, log rank $\chi^{2}=11.39$, $p=0.003)$. In multivariate analysis, Naa10p, SNCG, PRL-3, and TNM classification all remained independent prognostic factors for DMFS and OS (data not shown).

Prognostic Value of Multimarker System for Patients With Breast Cancer Stratified by and TNM classification

To explore whether our new classification system has the same prognosis value in patients with different TNM classification, all 365 patients were stratified in to Stage I subgroup $(n=141)$, Stage II subgroup $(n=128)$ and Stage 
Table 3. Independent Predictors of DMFS and OS in Multivariate Analysis of 365 Patients with Breast Cancer

\begin{tabular}{|c|c|c|c|c|}
\hline \multirow[b]{2}{*}{ Characteristics } & \multicolumn{2}{|c|}{ DMFS } & \multicolumn{2}{|c|}{ OS } \\
\hline & HR $(95 \%$ CI) & $p$ value & $\mathrm{HR}(95 \% \mathrm{CI})$ & $p$ value \\
\hline TNM stage & & $<0.001$ & & $<0.001$ \\
\hline I & 1 & & 1 & \\
\hline II & $1.780(1.021-3.103)$ & & $1.743(0.878-3.458)$ & \\
\hline III+IV & $8.313(4.988-13.856)$ & & $8.182(4.412-15.175)$ & \\
\hline Naa10p & & 0.001 & & 0.002 \\
\hline negative & 1 & & 1 & \\
\hline positive & $0.406(0.236-0.700)$ & & $0.344(0.172-0.686)$ & \\
\hline SNCG & & $<0.001$ & & 0.002 \\
\hline negative & 1 & & 1 & \\
\hline positive & $2.058(1.429-2.963)$ & & $1.977(1.294-3.019)$ & \\
\hline PRL-3 & & 0.037 & & 0.026 \\
\hline negative & 1 & & 1 & \\
\hline positive & $1.465(1.023-2.097)$ & & $1.596(1.057-2.409)$ & \\
\hline
\end{tabular}

*All factors that showed prognostic significance in the univariate Cox analysis were included in the multivariate Cox proportional hazard model. Only those clinicopathologic features and combined markers showing statistical significance $(p<0.05)$ are presented. HR, hazard ratio; CI, confidence interval.

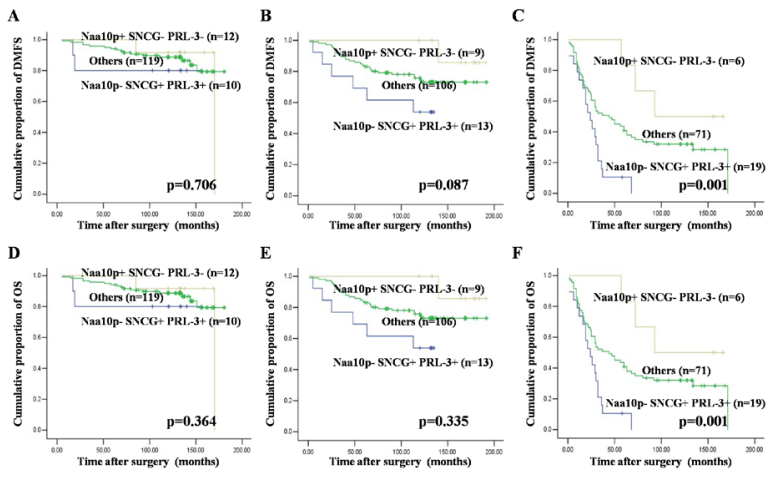

Figure 4. Kaplan-Meier Curve of DMFS and OS in 365 patients stratified with TNM Classification Evaluated According to Naa10p, SNCG, PRL-3 Combination. A) DMFS of Stage I; B) DMFS of Stage II; C: DMFS of Stage III/ IV; D: OS of Stage I; E) OS of stage II; F) OS of stage III/IV

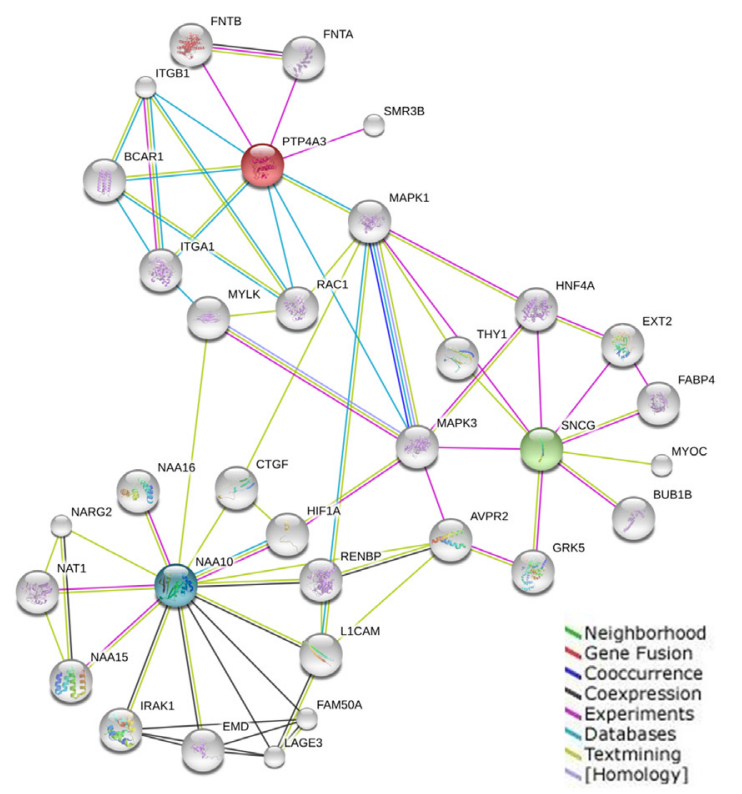

Figure 5. Gene-gene interaction networks of NAA10, SNCG, PTP4A3 and their related genes

III/IV (n=96) subgroup.

For patients of stage I and stage II, patients with Naa10p+SNCG-PRL-3- tumors had a trend of longer DMFS compared with other subgroups, but did not reach a statistical difference (Figure 4A, Stage I, log rank $\chi^{2}=0.696 ; p=0.706$; Figure 4B, Stage II, $\log \operatorname{rank} \chi^{2}=4.885$; $p=0.087)$. And for Stage III/IV patients, Naa10p+SNCGPRL-3- subgroup exhibited a significant longer DMFS than others (Figure $4 C$, long rank $\chi^{2}=15.034 ; p=0.001$ ). In regard of OS, our new classification system also performed better in Stage III/IV subgroup than in Stage II and Stage I subgroups (Figure 4D, Stage I, long rank $\chi^{2}=2.021$, $p=0.364$; Figure 4E, Stage II, long rank $\chi^{2}=2.186, p=0.335$; Figure $4 \mathrm{~F}$, long rank $\chi^{2}=14.367, p=0.001$ ).

In multivariate analysis, no variate was included in the model of DMFS or OS in Stage I subgroup. Only SNCG $[p=0.012$; HR $(95 \% \mathrm{CI})=2.401(1.213-4.753)]$ and Naa10p $[p=0.088 ;$ HR $(95 \% \mathrm{CI})=0.432(0.165-1.133)]$ remained independent prognostic factors for DMFS and only SNCG $[p=0.067$; HR $(95 \% \mathrm{CI})=2.150(0.948-4.876)]$ remained independent prognostic factors for OS in Stage II subgroup. For Stage III/IV patients, Naa10p [ $p=0.025$; HR $(95 \% \mathrm{CI})=0.405(0.184-0.891)]$, SNCG $[p=0.016$; $\operatorname{HR}(95 \% \mathrm{CI})=1.814(1.119-2.942)]$ and PRL-3 $[p=0.065$; HR $(95 \% \mathrm{CI})=1.570(0.972-2.535)]$ were all included in multivariate DMFS model, as well as in OS model [Naa10p, $p=0.016$; HR $(95 \% \mathrm{CI})=0.284(0.102-0.791)$; $\mathrm{SNCG}, p=0.057$; HR $(95 \% \mathrm{CI})=1.708(0.984-2.965)$; PRL$3, p=0.020 ; \mathrm{HR}(95 \% \mathrm{CI})=1.871(1.105-3.169)]$.

\section{Discussion}

Metastasis is the main cause of death in patients of breast cancer, and micrometastasis, the initial stage of metastasis has no reflection on patient's symptoms (Cox et al., 2011). Recently, micrometastasis is still hard to predict by any cancer biomarkers alone, resulting that patients at the same clinical stages often have different outcome (Jafferbhoy and McWilliams, 2012). In the current study, we have analyzed three molecules regulating cancer metastasis by different mechanisms, aiming to provide a more precise prediction of metastasis in breast cancer patients than the traditional molecular classification system consisting of ER, PR and HER2.

The components of our three biomarkers classification system are all involved in cell adhesion, motility and 
migration (Wang, 2006; Guo et al., 2007; Zeng et al., 2013). As showed in Figure 5, many of the molecules associated with Naa10p, SNCG and PRL-3 are metastasis-related proteins. Several key molecules, such as HIF-1 $\alpha$, integrin $\alpha 1$, integrin $\beta 1$, RAC1 and myocilin, were identified to be associated with the three biomarkers by STIRNG analysis (Franceschini et al., 2012). Even though there are some joint protein associated with 2 or 3 of Naa10p, SNCG and PRL-3, most of key molecules identified are just related to only one of the three biomarkers, meaning that these three biomarkers may represent different aspects of cancer metastasis mechanism.

Detailed information of published materials also supported this opinion. Though still under dispute, Naa10p was considered to have a very close relationship with HIF- $1 \alpha$ and involved in angiogenesis (Arnesen et al., 2005; Bilton, 2005; Jeong, 2002; Murrayrust et al., 2006). Naa10p also interacts with MLCK to repress cell motility (Bauer et al., 2009), interacts with PIX- $\beta$ to inhibit RAC1 pathway and to suppress metastasis of breast cancer cell (Hua et al., 2011). MMP2 and MMP9 were regulated by Naa10p (Zeng et al., 2013). Taken together, Naa10p had a negative role in breast cancer metastasis through several molecular pathways. For SNCG, which is involved in cell adhesion, invasion and metastases (Hibi et al., 2009; Pan et al., 2006), the molecular mechanism remained obscure. SNCG was also identified as an interacting protein of myocilin, which is believed to have a role in cytoskeletal function (Surgucheva et al., 2005). Therefore, it is not surprising that increased SNCG levels correlated with the presence of distant metastasis and unfavorable outcome. PRL-3 was found to promote cancer cell motility, invasion, and metastasis through integrin $\beta 1$-ERK1/2 and-MMP2 signaling (Ming et al., 2009; Peng et al., 2009; Vairaktaris et al., 2008). Based on these findings, we concluded that evaluation of all the three biomarkers expression level may provide a comprehensive view of different aspects of cancer metastasis mechanism, and may have a potential value in prognosis of breast cancer metastasis.

In this study, we revealed that both Naa10p, SNCG, PRL-3 alone and multimarker phenotype of Naa10p/ SNCG and of Naa10p/SNCG/PRL-3 were correlated with the presence of distant metastases, which also reflected in the postoperative survival time (DMFS and OS). Additionally, according to our results, molecular staging with three biomarkers (Naa10p, SNCG and PRL-3) identifies patient prognosis more accurately than the traditional clinical staging, particularly for patients with lymph node metastasis. The three biomarkers classification system also explained differences in the outcome of breast cancer patients belonging to the same TNM group, especially patients of Stage III and Stage IV. The reason why our new classification system performed better in patients with more advanced tumor were probably that all the three biomarkers were mainly conducive in the process of metastasis, which almost only occurred in cancer of late stage.

In conclusion, our results suggested that combined expression profile of Naa10p, SNCG and PRL-3, alone or in combination with TNM classification system, has better prognostic value for patients with breast cancer than the traditional molecular typing system, especially in patients with lymph node metastasis. The new multimarker system seemed to be promising in discriminating good from poor prognostic patients, with the potential to provide information for adjuvant therapy choice and, possibly, in establishing a more personalized prognosis model for each breast cancer patient.

\section{Acknowledgements}

This work was supported by the Doctoral Program of Higher Education of China (20110001110050) and National 973 Program of China (2009CB521805).

\section{References}

Al-Aidaroos A and Zeng Q (2010). PRL-3 phosphatase and cancer metastasis. J Cell Biochem, 111, 1087-98.

Amsterdam A, Shezen E, Raanan C, et al (2012). Differential staining of gamma synuclein in poorly differentiated compared to highly differentiated colon cancer cells. Oncol Rep, 27, 1451-4.

Arnesen T, Kong X, Evjenth R, et al (2005). Interaction between HIF-1 $\alpha$ (ODD) and hARD1 does not induce acetylation and destabilization of HIF-1 $\alpha$. FEBS Letters, 579, 6428-32.

Bauer J, Shin D, Chun Y, et al (2009). Arrest defective-1 controls tumor cell behavior by acetylating myosin light chain kinase. PLOS ONE, 4, 7451.

Bilton R (2005). Arrest-defective-1 protein, an acetyltransferase, does not alter stability of hypoxia-inducible factor (HIF)- $1 \alpha$ and is not induced by hypoxia or HIF. J Biol Chem, 280, 31132-40.

Cox C, Shamehdi C and Glancy J (2011). Sentinel node micrometastasis in breast cancer: clinically significant or not? Breast J, 17, 335-6.

Czekierdowski A, Czekierdowska S, Wielgos M, et al (2006). The role of $\mathrm{CpG}$ islands hypomethylation and abnormal expression of neuronal protein synuclein-gamma (SNCG) in ovarian cancer. Neuro Endocrinol Lett, 27, 381-6.

Franceschini A, Szklarczyk D, Frankild S, et al (2012). STRING v9.1: protein-protein interaction networks, with increased coverage and integration. Nucleic Acids Res, 41, 808-15.

Gromyko D, Arnesen T, Ryningen A, et al (2010). Depletion of the human $\mathrm{N} \alpha$-terminal acetyltransferase A induces p53dependent apoptosis and p53-independent growth inhibition. Int J Cancer, 127, 2777-89.

Guo J, Shou C, Meng L, et al (2007). Neuronal protein synuclein gamma predicts poor clinical outcome in breast cancer. Int J Cancer, 121, 1296-305.

Guzinska-Ustymowicz K, Kisluk J, Terlikowski S, et al (2013). Expression of phosphatase of regenerating liver-3 (PRL-3) in endometrioid cancer and lymph nodes metastases. $A d v$ Med Sci, 1, 7-12.

Guzinska-Ustymowicz K, Pryczynicz A, Kemona A, Ustymowicz M (2011). Immunohistochemical assessment of PRL-3 (PTP4A3) expression in tumor buds, invasion front, central region of tumor and metastases of colorectal cancer. $A d v$ Med Sci, 56, 39-43.

Hibi T, Mori T, Fukuma M, et al (2009). Synuclein-gamma is closely involved in perineural invasion and distant metastasis in mouse models and is a novel prognostic factor in pancreatic cancer. Clin Cancer Res, 15, 2864-71.

Holmes M, Chen W, Li L, et al (2010). Aspirin intake and survival after breast cancer. J Clin Oncol, 28, 1467-72.

Hua K, Tan C, Johansson G, et al (2011). N- $\alpha$-acetyltransferase 10 protein suppresses cancer cell metastasis by binding PIX 


\section{Li Min et al}

proteins and inhibiting Cdc42/Rac1 activity. Cancer Cell, 19, 218-31.

Inaba S, Li C, Shi Y, et al (2005). Synuclein inhibits the mitotic checkpoint function and promotes chromosomal instability of breast cancer cells. Breast Cancer Res Treat, 94, 25-35.

Jafferbhoy S, McWilliams B (2012). Clinical significance and management of sentinel node micrometastasis in invasive breast cancer. Clin Breast Cancer, 12, 308-12.

Jemal A, Bray F, Center M, et al (2011). Global cancer statistics. CA Cancer J Clin, 61, 69-90.

Jeong J, Bae M, Ahn M, et al (2002). Regulation and destabilization of HIF-1 alpha by ARD1. Cell, 111, 709-20.

Jia T, Liu Y, Liu J, et al (1999). Stimulation of breast cancer invasion and metastasis by synuclein gamma. Cancer Res, 59, 742-7.

Kuo H, Lee D, Chen C, et al (2010). ARD1 Stabilization of TSC2 suppresses tumorigenesis through the mTOR signaling pathway. Sci Signal, 3, 9.

Lee C, Ou D, Lee S, et al (2010). hNaa10p contributes to tumorigenesis by facilitating DNMT1-mediated tumor suppressor gene silencing. J Clin Invest, 120, 2920-30.

Lim J, Park J, Chun Y (2006). Human arrest defective 1 acetylates and activates $\beta$-catenin, promoting lung cancer cell proliferation. Cancer Res, 66, 10677-82.

Liu C, Dong B, Lu A, et al (2010). Synuclein gamma predicts poor clinical outcome in colon cancer with normal levels of carcinoembryonic antigen. BMC Cancer, 10, 359.

Min L, Xu H, Wang J, et al (2013). N- $\alpha$-acetyltransferase 10 protein is a negative regulator of $28 \mathrm{~S}$ proteasome through interaction with PA28 3 . FEBS Letters, 587, 1630-7.

Ming J, Liu N, Gu Y, et al (2009). PRL-3 facilitates angiogenesis and metastasis by increasing ERK phosphorylation and up-regulating the levels and activities of Rho-A/C in lung cancer. Pathol, 41, 118-26.

Murrayrust T, Oldham N, Hewitson K, et al (2006). Purified recombinant hARD1 does not catalyse acetylation of Lys532 of HIF-1 $\alpha$ fragments in vitro. FEBS Letters, 580, 1911-8.

Ohkawa N, Sugisaki S, Tokunaga E, et al (2008). $\mathrm{N}$-acetyltransferase ARD1-NAT1 regulates neuronal dendritic development. Genes Cells, 13, 1171-83.

Ohkawa N, Sugisaki S, Tokunaga E, et al (2007). ARD1-NAT1 complex regulates neuronal dendritic arborization through $\alpha$-tubulin acetylation. Neurosci Res, 58, 88 .

Pan Z, Bruening W and Godwin A (2006). Involvement of RHO GTPases and ERK in synuclein-gamma enhanced cancer cell motility. Int J Oncol, 29, 1201-5.

Peng L, Xing X, Li W, et al (2009). PRL-3 promotes the motility, invasion, and metastasis of LoVo colon cancer cells through PRL-3-integrin beta1-ERK1/2 and-MMP2 signaling. Mol Cancer, $8,110$.

Polato F, Codegoni A, Fruscio R, et al (2005). PRL-3 phosphatase is implicated in ovarian cancer growth. Clin Cancer Res, 11, 6835-9.

Polymeropoulos M, Lavedan C, Leroy E, et al (1997). Mutation in the gamma-synuclein gene identified in families with parkinson's disease. Science, 276, 20457.

Rahimzadeh M, Baghestani A, Gohari M, Pourhoseingholi M (2014). Estimation of the cure rate in Iranian breast cancer patients. Asian Pac J Cancer Prev, 15, 4839-42.

Saini K, Taylor C, Ramirez A, et al (2011). Role of the multidisciplinary team in breast cancer management: results from a large international survey involving 39 countries. Ann Oncol, 23, 853-9.

Seo J, Cha J, Park J, et al (2010). Arrest defective 1 autoacetylation is a critical step in its ability to stimulate cancer cell proliferation. Cancer Res, 70, 4422-32.

Siegel R, Naishadham D and Jemal A (2012). Cancer statistics,
2012. CA Cancer J Clin, 62, 10-29.

Singh V, Zhou Y, Marsh J, et al (2007). Synuclein-gamma targeting peptide inhibitor that enhances sensitivity of breast cancer cells to antimicrotubule drugs. Cancer Res, 67, 626-33.

Surgucheva I, Park B, Yue B, et al (2005). Interaction of myocilin with gamma-synuclein affects its secretion and aggregation. Cell Mol Neurobiol, 25, 1009-33.

Vairaktaris E, Yapijakis C, Serefoglou Z, et al (2008). Gene expression polymorphisms of interleukins- 1 beta, $-4,-6$, $-8,-10$, and tumor necrosis factors-alpha, -beta: regression analysis of their effect upon oral squamous cell carcinoma. J Cancer Res Clin Oncol, 134, 821-32.

Wang L (2006). Overexpression of phosphatase of regenerating liver-3 in breast cancer: association with a poor clinical outcome. Ann Oncol, 17, 1517-22.

Wang Z, He Y, Cai S, et al (2008). Expression and prognostic impact of PRL-3 in lymph node metastasis of gastric cancer: its molecular mechanism was investigated using artificial microRNA interference. Int J Cancer, 123, 1439-47.

Wu K, Huang S, Zhu M, et al (2013). Expression of synuclein gamma indicates poor prognosis of triple-negative breast cancer. Med Oncol, 30, 612.

Zeng Y, Liu C, Dong B, et al (2013). Inverse correlation between Naa10p and MMP-9 expression and the combined prognostic value in breast cancer patients. Med Oncol, 30, 562.

Zheng P, Liu Y, Chen L, et al (2010). Stathmin, a new target of PRL-3 identified by proteomic methods, plays a key role in progression and metastasis of colorectal cancer. J Proteome Res, 9, 4897-905. 